\title{
REFLEXÕES SOBRE O ENSINO DE GEOCIÊNCIAS NAS ESCOLAS DE TEMPO INTEGRAL DO ESTADO DE GOIÁS
}

\author{
ABOUT THE TEACHING GEOSCIENCES IN FULL-TIME SCHOOLS IN THE STATE OF GOIÁS
}

\author{
DANILO MISSIAS TEIXEIRA ${ }^{1}$, FÁBIO BRAZ MACHADO ${ }^{2}$, MARCELO MARTIN ZAFALON ${ }^{1}$
}

\begin{abstract}
1 Programa de Pós-graduação em Ensino e História de Ciências da Terra. Instituto de Geociências Universidade Estadual de Campinas
- UNICAMP. Rua Carlos Gomes, 250, Campinas - SP. E-mail: danquimica2010@gmail.com ; marcelozafalon@gmail.com

2 Universidade Federal do Paraná - UFPR, Campus Politécnico, Departamento de Geologia. Av. Cel. Francisco H. dos Santos, s/n Jardim das Américas, Curitiba - PR. Email: fabio.machado@ufpr.br
\end{abstract}

\begin{abstract}
Resumo - Promover o estudo de conceitos de Geociências na Educação Básica é um desafio para os professores, uma vez que não há espaço para este trabalho nos currículos escolares e encontra-se dificuldade para o diálogo entre as disciplinas de Ciências da Natureza e Geografia. As escolas comuns não oferecem muitas possibilidades para o estudo das Geociências o que destaca a necessidade de mais espaço e tempo, além de momentos que possibilitem o diálogo entre os professores de Química, Física, Biologia e Geografia. Isso poderia ser minimizado por meio de um período maior de ensino o que ocorre nos colégios de tempo integral. Nas escolas de tempo integral os estudantes possam mais tempo em sala de aula, com atividades diferenciadas e que possibilitam abordagens diversas. Assim, o objetivo deste trabalho é identificar as possibilidades e os desafios para o ensino de Geociências nas escolas de tempo integral do Estado de Goiás. As escolas de tempo integral do Estado de Goiás, assim como as das outras localidades do país, tem como base as principais disciplinas escolares, sendo estas definidas por meio da Base Nacional Comum Curricular (BNCC). A matriz curricular foi elaborada a partir do pressuposto de uma educação multidimensional onde são valorizados os conhecimentos científicos e as questões socioemocionais dos estudantes contemplada por meio dos componentes curriculares de duas partes: 1) Núcleo Comum; 2) Núcleo Diversificado. As possibilidades identificadas foram classificadas quanto a sua natureza em: Curricular, Pedagógica e Contextualização. Percebeu-se que, as disciplinas de Práticas de Laboratório e Pós-Medio se apresentam como espaços para o estudo de Geociências. O projeto de vida do aluno deve ser o principal objetivo numa escola integral e, por este motivo, os conceitos geocientíficos devem ser abordados de forma contextualizada, valorizando o contexto regional dos alunos e desenvolvendo no mesmo o pensamento crítico e a formação para o mercado de trabalho.
\end{abstract}

Palavras-chave: Ensino de Geociências,; Geoensino; Geodivulgação.

\begin{abstract}
Promoting the study of Geosciences concepts in Basic Education is a challenge for teachers, since there is no space for this work in school curricula and there is difficulty for the dialogue between the subjects of Natural Sciences and Geography. Regular schools do not offer many possibilities for the study of Geosciences, which highlights the need for more space and time, as well as moments that enable dialogue between teachers of Chemistry, Physics, Biology and Geography. This could be minimized through a longer period of teaching, which occurs in full-time colleges. In full-time schools, students can spend more time in the classroom, with different activities that allow for different approaches. The objective of this work is to identify the possibilities and challenges for the teaching of Geosciences in full-time schools in the State of Goiás. Full-time schools in the State of Goiás, as well as those in other locations in the country, are based on the main school subjects, which are defined through the Common National Curriculum Base (BNCC). The curricular matrix was elaborated from the assumption of a multidimensional education, where scientific knowledge and the students' socio-emotional issues are valued, contemplated through the curricular components of two parts: 1) Common Core; 2) Diversified Core. The identified possibilities were classified according to their nature, in: Curriculum, Pedagogical and Contextualization. It was noticed that the disciplines of Laboratory Practice and Post-Medium are presented as spaces for the study of Geosciences. The student's life project should be the main objective in an integral school. For this reason, geoscientific concepts must be approached in a contextualized way, valuing the regional context of the students, and must develop critical thinking and training for the labor market.
\end{abstract}

Keywords: Teaching of Geosciences; Geoteaching; Geodisclosure. 


\section{INTRODUÇÃO}

As Geociências na escola devem ser estudadas por meio da interação entre das disciplinas que compõem as Ciências da Natureza e a Geografia, o que direciona para o trabalho interdisciplinar.

Segundo Fazenda (2008) a interação entre disciplinas (interdisciplinaridade) propõe um estudo de maior complexidade sobre determinado fenômeno, sendo neste caso, os conceitos geocientíficos.

Ainda, a natureza não é compactada em sub partes, mas sim, em um sistema complexo, carregado de fenômenos e complexo para ser estudado. conforme destacado por Moura (2014) a natureza da Ciência é entendida como um conjunto de elementos que tratam da construção, estabelecimento e organização do conhecimento científico. Isto pode abranger desde questões internas, tais como método científico e relação entre experimento e teoria, até outras externas, como a influência de elementos sociais, culturais, religiosos e políticos na aceitação ou rejeição de ideias científicas.

Cabe ressaltar que a natureza é um conjunto de conhecimentos que transbordam o olhar, meramente disciplinar. Partindo desse pressuposto, pretende-se apresentar as contribuições das Ciências da Natureza para o entendimento de conceitos geocientíficos, o que, de fato, não é uma tarefa muito simples, uma vez que não se tem uma literatura científica muito ampla para esta discussão.

Assim, as escolas comuns não oferecem muitas possibilidades para o estudo das Geociências, por razões já discutidas. Todavia, a necessidade de mais espaço e tempo, além de momentos que possibilitem o diálogo entre os professores de Química, Física, Biologia e Geografia, podem ser minimizadas por meio de um período maior de ensino, o que ocorre nos colégios de tempo integral.

Nas escolas de tempo integral, os estudantes passam mais tempo no ambiente escolar e os professores possuem espaços para troca de ideias e planejamentos em coletivo.

As instituições de ensino que trabalham com a perspectiva de um número maior de aulas visam, dentre outros aspectos, proporcionar aos estudantes uma educação integral, ou seja, o estudo dos conceitos científicos, dos valores éticos e coletivos e das questões socioemocionais necessárias para um bom convívio em sociedade.

Nesse sentido, o objetivo deste artigo é identificar as possibilidades e os desafios para o ensino de Geociências nas escolas de tempo integral do Estado de Goiás. Para isso, será foi feita uma breve abordagem sobre a educação integral neste estado, estabelecendo os principais aspectos.

\section{ESCOLAS DE TEMPO INTEGRAL NO ESTADO DE GOIÁS}

Segundo o Centro de Referência em Educação Integral (EI) as escolas de tempo integral buscam uma educação baseada na formação de habilidades intelectuais, físicas, emocionais, sociais e culturais que são reflexo de um projeto coletivo elaborado entre gestores, professores, alunos, família e demais membros da comunidade a qual a instituição está presente.

Trata-se portanto de uma proposta contemporânea que atende as demandas do século XXI, que promove a inclusão dos diferentes indivíduos e equidade dos direitos educativos de todos os estudantes (EI, 2019).

Esta proposta de ensino deve proporcionar o estudo dos conceitos científicos de forma a contribuir na formação de sujeitos críticos e modificadores da sociedade. Seu currículo se baseia em duas grandes partes, a saber:

1) Base Comum;

2) Núcleo Diversificado.

A Base Comum compreende as disciplinas semelhantes às escolas de tempo parcial Matemática, Português, Química, Biologia, 
Geografia, dentre outras - e são responsáveis pelo estudo dos conceitos científicos "brutos" de quatro áreas de conhecimento: Matemática, Ciências da Natureza, Ciências Humanas e Linguagens.

A parte diversificada possibilita ao aluno o desenvolvimento de seu projeto de vida, por meio do diálogo com as disciplinas da Base Comum (INEP, 2009).

Assim, as escolas de tempo integral proporcionam espaço para os diferentes diálogos entre as disciplinas. É possível afirma, então, que os conceitos de Geociências - foco deste artigo - podem ser trabalhados durante as aulas destas intuições de ensino.

Uma terceira compreensão da educação integral a visualiza pela perspectiva da integração dos conhecimentos em abordagens interdisciplinares e transdisciplinares, focalizando especificamente o currículo escolar. A ênfase na articulação de conhecimentos e disciplinas que objetiva a integralização de experiências e saberes no processo educativo considera que as práticas educativas devem abrir-se as experiências e conteúdos transversais. Esta concepção de educação propõe uma estreita articulação curricular que contemple o conhecimento de maneira mais abrangente, global e, portanto, integral (INEP, 2009).

A partir desta afirmação, é possível identificar que o trabalho interdisciplinar (o diálogo entre as disciplinas) compõem a rotina das escolas de tempo integral. É possível ainda constatar quais as possibilidades para 0 estudo dos conceitos de Geociências nestas escolas, além de dos possíveis desafios.

Todavia, torna-se importante ressaltar que, as escolas de tempo integral são estruturadas (matriz curricular) de forma diferente. Todas elas mantêm a mesma essência, ou seja, possibilitar uma formação mais ampla e que abranja vários aspectos do indivíduo, mas as disciplinas que são trabalhadas irão variar de um estado para o outro.
Uma educação integral, entretanto, deve se basear em aspectos científicos, sociais, culturais e éticos, como definido pelo Ministério da Educação (MEC) e reafirmado no PNE. Isso perpassa por uma estrutura curricular diferenciada pensada com um projeto educativo integrado, em sintonia com a vida, as necessidades, possibilidades e interesses dos estudantes. Um projeto em que crianças, adolescentes e jovens são vistos como cidadãos de direitos em todas as suas dimensões. Não se trata apenas de seu desenvolvimento intelectual, mas também do físico, do cuidado com sua saúde, além do oferecimento de oportunidades para que desfrute e produza arte, conheça e valorize sua história e seu patrimônio cultural, tenha uma atitude responsável diante da natureza, aprenda a respeitar os direitos humanos e os das crianças e adolescentes, seja um cidadão criativo, empreendedor e participante, consciente de suas responsabilidades e direitos, capaz de ajudar o país e a humanidade a se tornarem cada vez mais justos e solidários, a respeitar as diferenças e a promover a convivência pacífica e fraterna entre todos (MEC, 2015).

Sendo assim, a escola em tempo integral deve proporcionar ao estudante o espaço para a construção do seu projeto de vida. Isso perpassa pelo ensino dos conceitos científicos, mas, também, pelo estímulo do diálogo, dos valores sociais e pela cidadania. São características também defendidas pelo Ensino de Geociências, sendo possível afirmar que existe uma perspectiva para esta área de conhecimento na estrutura curricular destas escolas.

\section{PERSPECTIVAS SOBRE O ENSINO DE GEOCIÊNCIAS}

Existem vários autores que pesquisam sobre as Geociências que buscam estabelecer definições e uma estruturação para esta área de conhecimento. Para Carneiro et al. (2004), as Geociências abordam aspectos que estabelecem uma relação entre as ciências naturais e humanas, além de promover o 
estudo do conhecimento científico numa perspectiva social e ambiental.

Partindo desta definição, torna-se possível identificar um discurso baseado em práticas que extrapolam a disciplinaridade, ou seja, os conceitos geocientíficos não pertencem a uma disciplina em si, mas a um conjunto de disciplinas. Isso pode ser observado em Compiani (2007) como aqueles conteúdos de Geologia, Meteorologia e alguns aspectos da Astronomia, que não constam como disciplina no currículo da escola fundamental, mas são abordados em Geografia e Ciências. Também incluí muitos conteúdos de Geociências que antes eram apenas tratados na Geografia Física e que hoje estão também em Ciências, tais como: Pedologia, Climatologia e Hidrologia.

O autor supracitado faz referência a conteúdos presentes em duas diferentes áreas de conhecimento: a Geografia e a Ciências da Natureza. Isso leva a pensar que a Geociências é o resultado do diálogo entre tais áreas de conhecimento, buscando identificar, estudar e apresentar resultados vinculados aos processos naturais e sociais do meio ambiente.

Também é possível afirmar, segundo colocações deste mesmo autor, que existe uma significação das Geociências como algo que se aproxima - quase como um sinônimo - da Geologia. Mas, para outros autores, estes dois conceitos não são iguais, apresentando aspectos bastante específicos para cada.

Hornink et al. (2013) afirmam que os conhecimentos geocientíficos são necessários na vida de todos os indivíduos, principalmente no que se refere às questões ambientais presentes no cotidiano. Os autores afirmam ainda que, tais conceitos, possuem forte influência dos conhecimentos das Ciências Biológicas, da Química, da Física e da Geografia.

Considera-se então Geociências como algo mais amplo e que abrange conhecimentos de disciplinas diferentes, mas que dialogam entre si. Nesse sentido, esta área de conhecimento deixa de ser, unicamente, um sinônimo da Geologia, como apontado em alguns referenciais. Essa afirmação vai de encontro ao que propõe Patapova (2016) quando o mesmo afirma que no estudo de um sistema natural integrado tal como a Terra, deveria haver uma ciência que sintetizasse conhecimentos sobre todas as formas de movimento da Matéria que tornam parte de uma evolução do sistema. Essa ciência é a Geologia, tomada no seu sentido mais amplo com a mais geral e ampla ciência do planeta.

O autor apresenta um conceito para a Geologia que se aproxima daquele apresentado por Hornink et al. (2013) para as Geociências. Observa-se a aparente referência à Química através do termo "matéria", principal fonte de estudo desta disciplina, o que leva a refletir que, as Geociências, muito embora sinonimizada à Geologia, se trata de um conceito mais amplo, abrangendo, inclusive, a própria Geologia.

Esta afirmação pode, ainda, ser ratificada por Viera et al. (2016), em que as Geociências, ou Ciências da Terra, envolvem todas as ciências que estudam o planeta Terra, incluindo geosfera, hidrosfera, atmosfera, biosfera.

É fato que os temas geocientíficos fazem parte dos Parâmetros Curriculares Nacionais (PCN1s), além de serem abordados em diversas ocasiões, como por exemplo, em programas e políticas de educação e para a formação de professores.

Nesse sentido, as Geociências são uma área de conhecimento, fruto do diálogo entre a Química, a Física, a Biologia (Ensino de Ciências) e a Geografia, englobando, também, a Geologia e outras ciências que estudam o Sistema Terra. Além disso, entender as Geociências possibilita subsídios para perceber o homem como um ser que é capaz de modificar o meio em que vive.

Mesmo tendo clareza sobre esta definição de Geociências, e da importância de seu estudo, 
ainda se mantém distante (ou não muito próxima) da área de Ciências da Natureza. Esta afirmação pode ser validada a partir do levantamento feito em Teixeira (2021) em se verificou que o número de trabalhos que tenham foco em conceitos geocientíficos, publicados por autores do Ensino de Ciências, ainda é muito pequeno.

Este distanciamento pode ser reflexo de uma necessidade de formação dos professores de Ciências da Natureza, no que diz respeito aos conceitos de Geociências. Para Teixeira et al. (2018), existe a necessidade de implantação destes conceitos nos cursos de formação dos docentes de Química, Física e Biologia, uma vez que, são estes profissionais que irão atuar no Ensino de Ciências na Educação Básica.

Ainda em Teixeira et al. (2018), foi feito um levantamento nos currículos de cursos de licenciatura em Biologia, Física e Química, em que buscou-se identificar a presença de disciplinas vinculadas a Geociências nas grades curriculares, em universidades dos estados da região Centro-Oeste do Brasil. O que identificou-se foi um número muito pequeno de matrizes curriculares que abordem os conceitos geocientíficos.

Sendo assim, fica evidente a necessidade de discutir mais sobre Geociências dentro da área do Ensino de Ciências. Já que, segundo os Parâmetros Curriculares Nacionais para o Ensino Fundamental e Médio preveem o estudo da Geologia, Mineralogia, Climatologia e demais campos da ciência que enfoquem no conhecimento do Sistema Terra e, consequentemente, das Geociências.

\section{IDENTIFICAÇÃO DAS POSSIBILIDADES E DESAFIOS}

As escolas de tempo integral do Estado de Goiás, assim como as das outras localidades do país, têm como base as principais disciplinas escolares - Matemática, Português, Geografia, Química, dentre outras. Sendo estas definidas, recentemente, por meio da Base Nacional Comum Curricular (BNCC).
A matriz curricular foi elaborada a partir do pressuposto de uma educação multidimensional, em que são valorizados os conhecimentos científicos e as questões socioemocionais dos estudantes.

Possui ainda uma carga horária de 9h30 diárias para o Ensino Médio, que contempla os componentes curriculares de duas partes: 1) Núcleo Comum; 2) Núcleo Diversificado. Apresentando, portanto, carga horária semanal de $45 \mathrm{~h} / \mathrm{a}$ semanais.

As disciplinas do Núcleo Comum são organizadas em quatro grupos, conforme apresentado no Quadro 1. Estão presentes nas escolas de tempo integral e, também, nas escolas de tempo parcial, sendo orientadas pela BNCC - a partir de sua implantação. São responsáveis pelo desenvolvimento cognitivo dos estudantes, através do ensino de conceitos científicos.

Quadro 01 - Estrutura Curricular das escolas de tempo integral do Estado de Goiás.

\begin{tabular}{|l|l|l|}
\hline \multicolumn{2}{|c|}{ NÚCLEO COMUM } & $\begin{array}{l}\text { NÚCLEO } \\
\text { DIVERSIFICADO }\end{array}$ \\
\hline \multirow{7}{*}{ Linguagens } & Português, & Prática de \\
& Educação & Laboratório \\
& Física, Língua & Avaliação \\
& Inglesa, & Semanal \\
& Língua & Preparação \\
& Espanhola, & Pós-Médio \\
& Arte. & Estudo \\
Ciências & Geografia, & Orientado \\
Humanas & História, & Projeto de Vida \\
& Sociologia, & Protagonismo \\
& Filosofia. & Juvenil \\
Ciências da da & Química, & Eletivas \\
Natureza & Física, & \\
\cline { 1 - 2 } Matemática & Biologia. & \\
\hline
\end{tabular}

O Núcleo Diversificado (ND) apresenta sete disciplinas, o que corresponde ao diferencial entre as escolas de tempo integral e as de tempo parcial, tanto em carga horária quanto em perspectivas pedagógicas. 
Segundo as orientações para o Modelo Pedagógico das Escolas Integrais do Estado de Goiás as disciplinas deste núcleo oferecem aos estudantes a exploração de capacidades cognitivas e sócioemocionais, uma vez que trabalha com os sonhos e habilidades do estudante.

No protagonismo juvenil, por exemplo, existem diversos clubes, como de xadrez, futebol, música, entre outros. São os próprios alunos que montam os clubes e que coordenam as atividades. Dessa forma, o estudante se sente parte da escola e protagonista de sua própria vida (Seduce, 2017).

As disciplinas do ND proporcionam o desenvolvimento de habilidades e conhecimentos através da articulação com o Núcleo Comum. Isso inclui o desenvolvimento de projetos científicos, culturais e artísticos, aulas contextualizadas e abordagens interdisciplinares, sendo visado o protagonismo dos estudantes.

Todas estas disciplinas buscam proporcionar aos estudantes subsídios para a elaboração do seu Projeto de Vida, que inclui seus sonhos, valores, dificuldades, metas a serem traçadas e objetivos a serem alcançados.

Os conhecimentos científicos devem ser mediados de forma que apresentem sentido para o estudante, ou seja, os conceitos devem contribuir para o seu projeto de vida. Nesse sentido, os conceitos geocientíficos devem ser trabalhado em sala de aula com base nesse propósito.

Por meio da breve descrição sobre as escolas de tempo integral, torna-se possível identificar as possíveis maneiras de se promover o estudo de conceitos de Geociências nesses espaços. Essas possibilidades estão relacionadas a três naturezas conforme demonstra a Fig. 1, a saber:

1) Curricular - possiblidades relacionadas à estrutura curricular;
2) Pedagógica - quando está relacionada ao modelo pedagógico da escola, e suas atividades práticas;

3) Contextualização - que relaciona as possibilidades relacionadas aos conteúdos das disciplinas do Núcleo Comum.

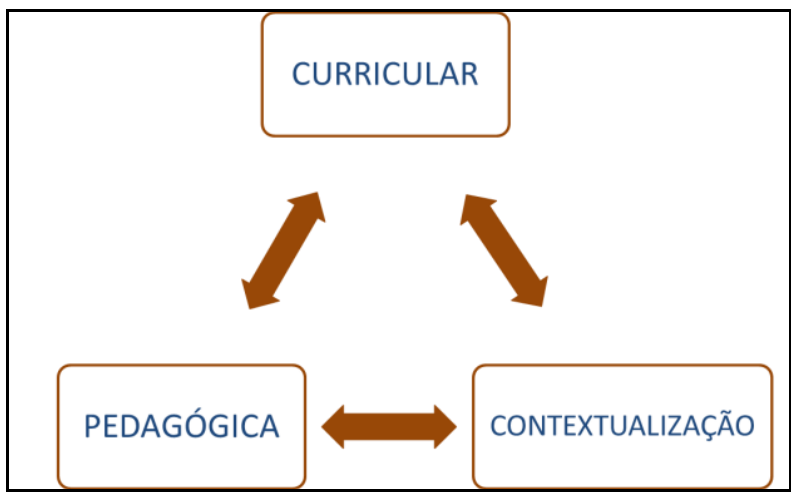

Figura 1. Natureza das possibilidades para o estudo de Geociências nas escolas de tempo integral.

A estrutura curricular possibilita o trabalho interdisciplinar dos conceitos científicos, bem como a interação entre as disciplinas do Núcleo Diversificado (ND) e do Núcleo Comum (NC).

Por meio disso torna-se possível afirmar que os conceitos de Geociências podem ser trabalhados sob essa perspectiva, uma vez que, como proposto por Hornink; Galembeck; Compiani (2013) tais conceitos tem natureza interdisciplinar, sendo abordados pelo diálogo entre diferentes disciplinas escolares.

A interação entre os dois núcleos de conhecimentos das escolas de tempo integral possibilita a articulação de vários conhecimentos, como destacado no Modelo Pedagógico. As disciplinas do ND podem ser utilizadas como um espaço para aplicação prática dos conceitos trabalhados nas aulas de disciplinas do NC, o que nos direciona à natureza PEDAGÓGICA destes conceitos.

Nesse sentido, tanto a matriz CURRICULAR quanto a parte PEDAGÓGICA devem possibilitar o estudo de Geociências na escola. Para Carneiro et al. (2004), estudar os conceitos geocientíficos na escola traz questões atuais e relevantes vinculadas à 
sustentabilidade e o cuidado com o meio ambiente. É, portanto, um momento que direciona o aluno a adquirir conhecimentos de mundo.

Costa (2016) reforma este pensamento quando afirma que o conhecimento é produzido a partir de uma construção coletiva e este conhecimento historicamente acumulado deve se colocar a serviço da transformação social que se apresenta pelas vivências e experiências. E, desta forma, a Educação Integral é possibilitada por uma busca que tem a realidade, as vivências e as experiências, que não se fragmentam, como motor para esta empreitada.

Nesse sentido, a importância do contexto social é inquestionável, uma vez que, é por meio dela que se permite ao aluno a formação do senso crítico e a atual dentro de sua própria realidade. Isso leva esta discussão para a terceira natureza destacada, a CONTEXTUALIZAÇÃO.

Assim, pode-se afirmar que, as naturezas para o trabalho dos conceitos de Geociências nas escolas de tempo integral estão relacionadas entre si, compondo um conjunto ideal para este trabalho.

Todavia, na prática, o que se observa é o estudo dentro das disciplinas escolares. Como já destacado neste artigo, o projeto de vida do aluno é o foco central das escolas de tempo integral. Sendo assim, os conceitos geocientíficos devem contribuir para que este objetivo seja alcançado. A Fig. 02 apresenta a relação das disciplinas escolares em direção ao projeto de vida do aluno.

O trabalho interdisciplinar pode ser feito a partir da interação entre as disciplinas do ND e do NC, levando em consideração o contexto social do aluno. As disciplinas de Práticas de Laboratório e Pós-Médio são aquelas que mais se aproximam dessa possibilidade, uma vez que suas ementas visam estabelecer momentos de vivências práticas para os alunos.

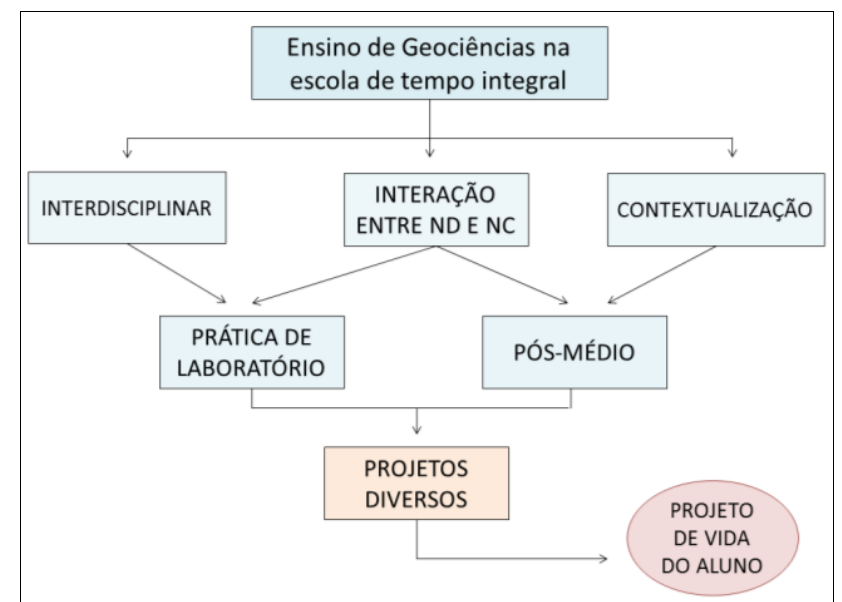

Figura 02. Disciplinas da escola integral que possibilitam o estudo de conceitos de Geociências.

As Práticas de Laboratório devem visar o estudo das disciplinas de Ciências da Natureza (Química, Física e Biologia) e da Matemática, utilizando o Laboratório de Ciências como espaço pedagógico (Seduce, 2017). Essa colocação disposta no Modelo pedagógico das escolas de tempo integral do estado de Goiás dialoga muito bem com a perspectiva apresentada pelos autores da área de Geociências (Hornin; Galembeck; Compiani, 2013; Ernesto et al., 2018), sendo esta disciplina uma possibilidade para o estudo dos conceitos desta área de conhecimento.

A dificuldade, muitas vezes, se encontra no material para execução das aulas práticas, bem como na condução das aulas pelos professores.

Andrade e Massabni (2011) justificam não utilizarem atividades práticas em suas aulas, sobretudo, por causa do grande número de alunos por turma, dificuldade esta que não depende só delas para ser superada. A necessidade de providenciar, com a direção, ou mesmo com os alunos, o material para as atividades práticas indica que estes materiais não estão prontamente disponíveis para a utilização e que, possivelmente, não há ou não se usa um local de armazenagem, como um laboratório. Assim, esta indisponibilidade 
de material e local é um fator para a nãoutilização de atividades práticas.

Sendo assim, para que as aulas de Práticas de Laboratório possam ser utilizadas para o estudo de conceitos de Geociências, torna-se necessário que haja material e um local apropriado disponível para as aulas sejam bem sucedidas. Com estas condições, as aulas experimentais podem ser um recurso interessante para trabalhar, na prática, os fenômenos geocientíficos.

Em relação à disciplina de Pós-Médio, não há na literatura um material que possa auxiliar na discussão desta disciplina. Segundo o Modelo Pedagógico das escolas de tempo integral do estado de Goiás, o pós-médio tem o objetivo de promover a discussão de questões atuais que possam auxiliar na sobrevivência dos estudantes no meio social, em seu ingresso no mercado de trabalho e na elaboração e implementação de seu projeto de vida (Seduce, 2017).

Para Alves (2016), o pós-médio pode ser utilizado como um espaço para o desenvolvimento do protagonismo e da cidadania. Isso inclui o entendimento da natureza e da relação do homem com o meio ambiente, suas modificações e a exploração dos recursos naturais. Assim, as aulas de pósmédio podem ser utilizadas como momento para promover essas discussões.

A grande dificuldade do pós-médio é a falta de material de apoio pedagógico que defina, de forma mais precisa, esta disciplinas. Segundo Alves (2016 p.29) o pós-médio é uma "disciplina ofertada apenas no último ano de ensino médio. Tem o objetivo de preparar os estudantes para a vivência após o ensino médio seja para o ingresso no ensino superior ou no mercado de trabalho". Essa definição abre um grande espaço de discussão, gerando inúmeros possibilidades de estudo, o que demanda uma lista de conceitos que devem ser primordiais na disciplina.

Tanto as Práticas de Laboratório quanto o Pós-Médio podem proporcionar espaço para professores e alunos elaborarem Projetos Diversos, com o intuito de discutir e divulgar conhecimento no ambiente escolar. Os conceitos de Geociências podem ser contemplados nestes projetos.

Os projetos devem possibilitar a elaboração e o enriquecimento do Projeto de Vida dos estudantes. Existe uma necessidade de se incentivar os estudantes e apoiá-los no processo de reflexão sobre "quem ele sabe que é" e "quem ele gostaria de vir a ser" e ajudá-lo a planejar o caminho que precisa construir e seguir, para realizar esse "encontro". Dessa forma, ao final do ensino médio, cada jovem deverá ter, minimamente, traçado aquilo que deseja construir nas dimensões pessoal, social e produtiva da vida, ou seja, o projeto da sua vida.

Nas aulas de Projeto de Vida, os estudantes são levados a refletir sobre sua relação com o mundo, o que será importante em sua atuação na sociedade, por meio da tomada de decisões e de suas ações. O respeito ao meio ambiente, o cuidado com os espaços sociais e o desenvolvimento de valores são conceitos trabalhados nestas aulas.

Nesse sentido, o estudo dos conceitos de Geociências nas escolas de tempo integral do estado de Goiás deve proporcionar o desenvolvimento do Projeto de Vida do estudante. As aulas de Pós-Médio e Práticas de Laboratório, bem como os Projetos a serem desenvolvidos nestas escolas, são possibilidades para o estudo dos conceitos geocientíficos e para despertar o protagonismo.

\section{CONSIDERAÇÕES FINAIS}

As escolas de tempo integral do Estado de Goiás, assim como as demais unidades brasileiras, têm como principal foco de ensino o projeto de vida dos estudantes. Nesse contexto, entende-se que os conceitos de Geociências devem ser abordados de forma a possibilitar a construção deste projeto de vida, estabelecendo sentido ao ensino promovido em sala de aula. 
Após a análise da estrutura curricular destas instituições foi possível identificar possibilidades dentro de três dimensões: Curricular, Pedagógica e Contextualização. Percebeu-se que, as disciplinas de Práticas de Laboratório e Pós-Medio se apresentam como espaços para o estudo de Geociências.

Estas dimensões dialogam entre si, o que estabelece um material de análise bastante rico e, além disso, proporciona que os conceitos de Geociências sejam abordados e aprendidos de forma didática, mediada e contextualizada. Assim, estas possibilidades caracterizam as escolas de tempo integral como locais propícios para estudar conteúdos geocientíficos.

\section{AGRADECIMENTOS}

Danilo Missias Teixeira e Marcelo Martin Zafalon agradecem a Coordenação de Aperfeiçoamento de Pessoal de Nível Superior (CAPES) pela bolsas de pósgraduação.

\section{REFERÊNCIAS}

ALVES, N. O sentido da escola. Rio de Janeiro: DP\&A, 2016.

ANDRADE, M. L. F.; MASSABNI, V. G. O desenvolvimento de atividades práticas na escola: um desafio para os professores de ciências. Ciência \& Educação, v. 17, n. 4, p. 835-854, 2011.

CARNEIRO, C. D. R.; TOLEDO, M. C. M. e ALMEIDA, F. F. M.. Dez motivos para inclusão de temas de Geologia na Educação Básica. Revista Brasileira de Geociências, v. 34, p. 553-560, 2004.

COMPIANI, M. O lugar e as escadas e suas dimensões horizontal e vertical nos trabalhos práticos: implicações para o ensino de Ciências e educação ambiental. Ciência \& Educação, v.13, n.11, p.29-45, 2007.

COSTA, N. Cenário da Educação Integral no Brasil. 2016. Disponível em: $<$ https://educacaointegral.org.br/reportagens /como-sera-cenario-da-educacao-integralem-2016/> Acesso em 04 junho 2020.

El - Centro de Referência em Educação Integral. 2019. Disponível em: <https://educacaointegral.org.br/conceito/> Acesso 22 jun 2020.

HORNINK, G. G.; GALEMBECK, E; COMPIANI, M. Traçando caminhos ao "Geociências virtual" - Reflexões sobre comunidades online de aprendizagem. Revista Terrae Didática. v.9. p. 22-33, 2013.

INSTITUTO NACIONAL DE ESTUDOS E PESQUISAS EDUCACIONAIS ANÍSIO TEIXEIRA (INEP). Censo Escolar, 2009. Brasília: MEC, 2011. JANUZZI, Paulo.

Ministério da Educação/SETEC. Currículo Referência: políticas públicas para a educação profissional e tecnológica. Brasília: MEC, 2004. BRASIL.

MOURA, B. A. O que é natureza da Ciência e qual sua relação com a História e Filosofia da Ciência?. Revista Brasileira de História da Ciência. v.7, n.1, p.32-46, 2014.

PATAPOVA, M. S. Geologia como uma ciência histórica da natureza. Revista Terrae Didática. v.3. p. 86-90. 1968.

SECRETARIA DE ESTADO DE EDUCAÇÃO DE GOIÁS, SEDUCE. 2017. Disponível em: $<$ http://homologacaosite.seduce.go.gov.br/po rtalseduce/ensino-integral/> Acesso em 22 de jun 2020.

TEIXEIRA, D. M. A interdisciplinar para o ensino de conceitos de geociências. 2017. Tese (Doutorado em Ensino e História de Ciências da Terra) - Universidade de Campinas, Coordenação de Aperfeiçoamento de Pessoal de Nível Superior.

TEIXEIRA, D. M.; MACHADO F. B., SILVA J. S.. O lúdico e o ensino de Geociências no Brasil: principais tendências das publicações na área de Ciências da Natureza. Revista Terrae Didatica. v.13, n.03, p. 286-294, 2018.

Submetido em 06/10/2021 Aceito em 06/12/2021 